
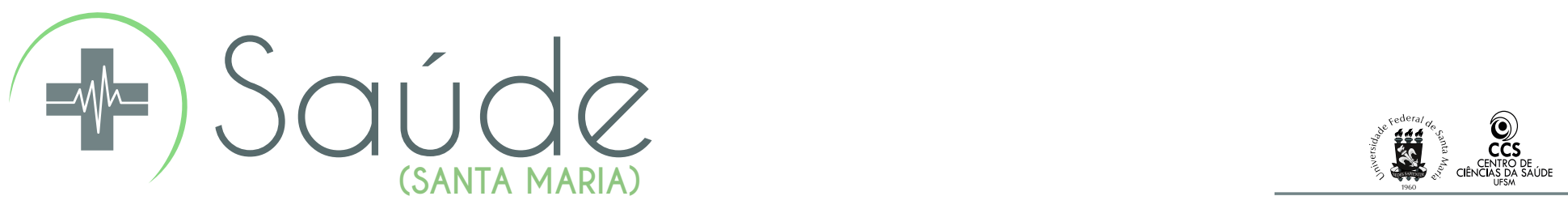

Nutrição

DOI: $10.5902 / 2236583425334$

\title{
Avaliação da efetividade de boas práticas em Serviços de Alimentação Coletiva em uma capital brasileira
}

\section{Effectiveness evaluation of good practices in Collective Food Services in a Brazilian capital}

Jaqueline Menti Boff, Virgílio José Strasburg

\section{RESUMO}

Objetivou-se verificar o grau de adequação de registros para a garantia higiênico sanitária dos alimentos em Serviços de Alimentação Coletiva (SAC). Metodologia: Adaptação de checklist pela Portaria SES/RS n 78/2009 com 34 itens, relativo à comprovação dos procedimentos em SAC: Institucionais Públicos, Privados de Buffet /quilo e Lanchonetes. As visitas aos SAC ocorreram em 2015, em Porto Alegre/RS. Os dados foram apurados no software Microsoft Office Exce/ , sendo classificados conforme a RDC 275/2002. Resultados: Participaram 15 estabelecimentos, distribuídos igualitariamente em cada modalidade. Os restaurantes públicos e privados atingiram um nível de adequação regular. $O$ percentual de adequação no total dos locais ficou em $60,5 \%$. 0 manejo de resíduos apresentou adequação de 93,3\%, enquanto o de edificação e instalações teve o menor percentual (55,5\%). Conclusão: A correta utilização de registros e o monitoramento desses podem auxiliar na prevenção de inconformidades e, consequentemente, de enfermidades relacionadas com os processos na manipulação dos alimentos.

Descritores: Documentação; Boas práticas de manipulação; Legislação sobre alimentos.

\begin{abstract}
Autor correspondente:
Jaqueline Menti Boff

E-mail: jak535@hotmail.com

Telefone: (5 I) 99909.5574

Formação Profissional: Formada

em Nutrição pela Universidade

Federal do Rio Grande do Sul

(UFRCS); Porto Alegre, RS, Brasil.

Filiação Institucional: UFRGS

Endereço para correspondência:

Rua: Ramiro Barcelos, 2400, $4^{\circ}$

andar, sala Nut 1, bairro Santa

Cecilia, Porto Alegre, RS, CEP:

90035-003
\end{abstract}

Data de Submissão: 23/12/2016

Data de aceite: 08/02/2018

Conflito de Interesse: Não há conflito de interesse.

\begin{abstract}
The objective was to verify the level of adequacy for registers for sanitary hygienic guarantee of food in Collective Food Services (CFS). Methodology: Adaptation of checklist from the Decree SES/RS no 78/2009 with 34 items, regarding the proof of procedures in CFS: Public Institutions, Private Buffet/kilo and Snack Bar. The visits to the CFS occurred in 2015, In Porto Alegre/RS. The

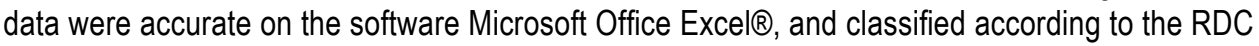
275/2002. Results: Participated 15 establishments, equally distributed in each modality. Public and private restaurants have achieved a regular adequacy level. The percentage of adequacy in all of the places was $60.5 \%$. The waste management presented a $93.3 \%$ adequacy, while the building and installations had the lowest percentage (55.5\%). Conclusion: The correct use of registers and monitoring of these can assist in the prevention of nonconformities and consequently diseases related to the processes in food handling.
\end{abstract}

Descriptors: Documentation; Good manipulation practices; Food legislation.

\section{(c) BY-NC-ND}




\section{Introdução}

O conceito de Segurança Alimentar e Nutricional (SAN) engloba duas acepções. Uma sendo no sentido alimentar e de acesso aos alimentos ${ }^{1}$ e a outra, referente ao contexto higiênico sanitário, que pode ser entendida como um conjunto de propriedades dos alimentos que resultam de sua inocuidade2. Assim, implica na ausência de qualquer tipo de risco de contaminação dos alimentos para consumo humano3. 0 aumento da população nos grandes centros urbanos, associada à participação das mulheres no mercado de trabalho e as alterações no estilo de vida, vem induzindo mudanças nos hábitos e costumes alimentares das pessoas ${ }^{4}$. Logo, a relação saúde-doença é diretamente proporcional à alimentação equilibrada e ao controle higiênico-sanitário dos alimentos 5 .

Como a rotina das pessoas está mudando, um número crescente de brasileiros começou a comer fora de suas casas, procurando por restaurantes, cafés, padarias e outros estabelecimentos ${ }^{6}$. No ano de 2015 , foi estimada a quantidade de 18,78 milhões de refeições por dia realizadas por empresas nas modalidades de autogestão, prestadoras de serviço e de restaurantes que trabalham com tíquetes convênio ${ }^{7}$.

Assim, a ausência de um controle rigoroso no processamento, no armazenamento e na distribuição dos alimentos apresenta um grande potencial para se tornar uma fonte importante de Doenças Transmitidas por Alimentos (DTAs)8. Estas podem ser causadas pela presença de toxinas produzidas por micro-organismos presentes nos alimentos ${ }^{9}$. Desta forma, as doenças causadas são provenientes de bebidas ou alimentos que sofreram alguma contaminação prévia10.

As DTAs são um problema persistente que continua a afligir as pessoas ao redor do mundo ${ }^{11}$. A cada ano, nos Estados Unidos da América, estima-se que existam 9.4 milhões de episódios de doenças transmitidas por alimentos, 55,961 mil hospitalizações e 1.351 mortes aproximadamente11. As DTAs também acabam por constituir um grande problema de saúde pública no Brasil, sendo responsáveis por elevados gastos econômicos e sociais ${ }^{12}$. Entre os anos de 2000 a 2013 ocorreram 8.871 surtos de DTAs, porém não refletem a realidade, uma vez que nem todos os casos são adequadamente notificados ${ }^{13}$. Uma diminuição de gastos com medicamentos, internações e perdas de dias de trabalho, e outras perdas como as sociais poderiam ser resolvidas com a diminuição do número desses surtos ${ }^{10}$.

Porto Alegre é um município brasileiro, pertencente ao estado do Rio Grande do Sul e também a capital mais ao sul do Brasil. É constituída por uma população de aproximadamente 1.409.351 habitantes, e possui índice de Desenvolvimento Humano (IDH) de 0.865, segundo dados de 2010 do Instituto Brasileiro de Geografia e Estatística (IBGE)14. Estima-se que o município tenha em média quatro mil locais para alimentação, como restaurantes, lanchonetes, bares e similares15.

A RDC 216 de 200416, da Agência Nacional de Vigilância Sanitária (ANVISA), estabelece que todos os locais que produzem e manipulam alimentos devem aplicar procedimento de boas práticas, visando à segurança do produto final 17 . Por sua vez, no Rio Grande do Sul, a Portaria $n^{0} 78$ de 2009 da Secretaria Estadual da Saúde considera a necessidade constante de aperfeiçoamento das ações de controle sanitário na área de alimentos ${ }^{18}$.

Sendo assim, a preocupação com a qualidade dos alimentos tem crescido e diversas ferramentas de gestão da qualidade têm sido criadas e utilizadas para oferecer um produto com qualidade até o momento em que será consumido ${ }^{19}$.

Diante da importância em assegurar a sanidade dos alimentos ofertados para o consumo da população, o presente estudo teve como objetivo verificar o grau de adequação dos registros sanitários preconizados na legislação, em três diferentes modalidades de Serviços de Alimentação Coletiva (SAC) em Porto Alegre/RS.

\section{Metodologia}

Foi realizado um estudo transversal descritivo, de caráter aplicativo e observacional, com amostra por conveniência, sendo realizado em três tipos de modalidades em SAC, na cidade de Porto Alegre, RS. Os restaurantes foram buscados através de consulta na internet, no qual foi possível localizar os três tipos de modalidade de SAC na cidade. A amostra selecionada resultou em 15 locais, divididos de forma igualitária (cinco) para cada modalidade de SAC: restaurantes institucionais públicos, restaurantes privados na modalidade de buffet livre ou por quilograma e lanchonetes. Foram visitados aqueles que estavam mais próximos e eram de mais fácil acesso para os pesquisadores. A visita em cada local foi realizada entre janeiro e março de 2015, sendo o primeiro contato realizado por ligações telefônicas, no qual foi explicado o motivo da pesquisa. A partir deste primeiro contato, ficava agendado um dia para que fosse possível realizar a visita conforme a disponibilidade de cada local. 0 critério de inclusão para a pesquisa era se o estabelecimento possuía nutricionista própria ou contratada. Foi desenvolvido um checklist adaptado da Portaria n 78/2009 SES/RS18, 
no qual foram selecionados 34 itens relativos às condições higiênico-sanitárias.

Os itens escolhidos correspondem à documentação que comprovem a execução correta dos procedimentos obrigatórios divididos em oito grupos: 1) Edificações, instalações, equipamentos, móveis e utensílios; 2) Higienização de instalações, Controle integrado de pragas e Abastecimento de Água; 3) Manejo de resíduos; 4) Manipuladores, Matérias primas, ingredientes e embalagens; 5) Preparação do alimento; 6) Armazenamento e transporte do alimento preparado; 7) Exposição ao consumo do alimento preparado; e 8) Documentação e registros. A forma de preenchimento do checklist foi dividido por "Adequado", "Inadequado" e "Não se aplica", quando determinado item não fosse pertinente a rotina do local.

Após a coleta das informações os dados foram calculados no software da Microsoft Office Excel囚 e analisados de forma descritiva para cada local e por modalidade de SAC. Dos resultados obtidos foram desenvolvidas tabelas separadas pela pontuação em três escalas. A classificação seguiu os padrões estabelecidos na RDC n⿳275/200220: a) Ruim - de zero até 50\% itens adequado; b) Regular - de 51\% a 75\% de adequação; e c) Ótimo - para conformidade de 76 a $100 \%$.

Os locais que aceitaram o convite para participar tiveram total sigilo de seus nomes e outros possíveis dados, assegurados por um Termo de Consentimento Livre e Esclarecido específico que foi entregue antes de participarem da pesquisa, ficando uma cópia para o lugar e outra assinada pela nutricionista ou responsável técnico para os pesquisadores. A Comissão de Pesquisa da Faculdade de Medicina da Universidade Federal do Rio Grande do Sul (UFRGS) aprovou 0 projeto referente a este estudo sob no: 27971/2014.

\section{RESULTADOS E DISCUSSÃO:}

A Tabela 1 apresenta os dados referentes à classificação geral e por modalidade de SAC (públicos, privados e lanchonetes) dos 15 estabelecimentos visitados conforme estabelecido pela Resolução da Diretoria Colegiada RDC $n^{\circ}$ 275/200220. Pode ser observado que as classificações de "ruim", "regular" e "ótima" se deu através da pontuação gerada nos checklists aplicados nos locais.

Tabela 1 - Classificação dos SAC visitados quanto à pontuação gerada de acordo com os critérios da RDC $n^{0}$ 275/2002. Porto Alegre, RS, 2015.

Pontuação (\%)

\begin{tabular}{lccc}
\hline Locais (n) & Ruim \% $(\mathrm{n})^{*}$ & Regular \% $(\mathrm{n})^{*}$ & Ótima \% $(\mathrm{n})^{*}$ \\
Geral (15) & $26,7(4)$ & $46,6(7)$ & $26,7(4)$ \\
Públicos (5) & $20(1)$ & $60(3)$ & $20(1)$ \\
Privados (5) & $0(0)$ & $80(4)$ & $20(1)$ \\
Lanchonetes(5) & $60(3)$ & $0(0)$ & $40(2)$ \\
\hline
\end{tabular}

$\%(n)^{*}$ : Ruim (0 - 50\%); Regular (51 - 75\%); Ótima $(76-100 \%)$;

Assim, foi possível observar que a classificação geral de todos os locais participantes da pesquisa se deu como regular, enquanto que quando divididos em suas categorias, os SAC públicos e privados também apresentaram uma resposta geral como regulares, tendo resultados de 60 e $80 \%$. Já as lanchonetes na sua maioria foram classificadas como ruins. Akutsu et al.21, em seu estudo com Unidades de Alimentação e Nutrição também encontraram $80 \%$ dos locais pesquisados no grupo II (denominado assim por ter de 30 a 69,9\% de atendimento a itens imprescindíveis). Já para Santos et al. ${ }^{22}$ na sua pesquisa realizada em cinco restaurantes de pequeno e médio porte na cidade do Rio de Janeiro, RJ, a média dos percentuais de adequação dos estabelecimentos em relação aos itens avaliados foi de 43,6\%.

Na modalidade das lanchonetes foi possível observar que $60 \%$ dos locais visitados tiveram a sua classificação ruim, ou seja, até 17 itens dos 34 presentes no checklist aplicado classificados como inadequados. Um resultado semelhante também foi encontrado por Batista et al.24, na qual duas lanchonetes foram avaliadas, sendo que ambos locais tiveram a sua classificação insatisfatória. Estes locais podem ter apresentado um resultado diferente do encontrado nas outras modalidades por apenas dois locais terem a figura da nutricionista presente diariamente e nos outros três apenas uma consultora de nutrição. 
A Tabela 2 apresenta os dados referentes a pontuação do total de itens adequados e inadequados dos locais. Assim, tais resultados foram separados conforme cada modalidade de SAC, através da contagem de 34 itens do checklist. 0 total apresentado no final da tabela refere-se aos 15 estabelecimentos, sem separações por modalidade de restaurante.

Tabela 2 - Pontuação total de itens adequados e inadequados dos SAC. Porto Alegre, RS, 2015.

\begin{tabular}{lccc}
\hline \multicolumn{3}{c}{ Pontuação (\%) } \\
\hline Locais & Adequado & Inadequado & Não se Aplica \\
\hline Públicos & 62,4 & 31,1 & 6,5 \\
Privados & 62,9 & 27,1 & 10 \\
Lanchonetes & 56,5 & 29,4 & 14,1 \\
Média & 60,6 & 29,2 & 10,2 \\
\hline
\end{tabular}

Desta maneira, observa-se na tabela 2 que nos restaurantes públicos e privados o percentual de adequação foi o mesmo de $63 \%$. Já Bernardo et al.25, com sua pesquisa realizada em hotéis de Belo Horizonte constatou que $60 \%$ dos estabelecimentos cumpriram de $76 \%$ a $100 \%$ dos itens imprescindíveis da lista de verificação, tendo uma ótima classificação contra $40 \%$ que atenderam de $51 \%$ a $75 \%$ dos itens, sendo sua classificação regular.

Ainda assim a média total dos locais participantes da pesquisa se mostrou dentro do nível regular de adequação, dentro de $60,5 \%$, que, segundo Akutsu et al.21 pode ser influenciado positivamente no preenchimento dos itens do checklist devido à presença de um responsável técnico, podendo ser a nutricionista ou gerente do local.

Todos os três tipos de restaurantes apresentaram resultados similares, porém as lanchonetes apresentaram um grau de inadequações superior aos restaurantes privados, podendo ser influenciada ou não ao investimento feito nestes locais. No entanto, apesar das lanchonetes participantes apresentarem menor percentual de adequação dos itens do checklist, obtiveram os maiores resultados de atividades não realizadas, ou seja, o item "Não se aplica". Isto se deve ao fato desses locais terem uma menor variedade de alimentos, subsequente a menor manipulação dos mesmos e menos registros gerados, quando comparadas aos demais SAC. Um estudo realizado em restaurantes do tipo self-service em Pernambuco17 encontrou que todos os estabelecimentos avaliados foram inadequados em relação à documentação $\mathrm{e}$ registros exigidos na legislação, sendo o alvará de funcionamento o único presente nos locais. Bernardo et al. 25 também encontrou no seu estudo o menor percentual referente à documentação $(45,2 \%)$.

Conforme descrito na tabela 3, os 34 itens do checklist foram separados em oito grupos para serem avaliados. 0 grupo de maior adequação, com $93,3 \%$ dos itens adequados, foi o de manejo de resíduos (3). Enquanto o de edificações, instalações, equipamentos, móveis e utensílios (1) foi o que apresentou maior inadequação, representando $55,5 \%$ do total de locais.

Esse maior percentual de inadequação já foi observado em outros estudos realizados, onde Campos et al.17 refere à ausência da prática de registros que comprovem a manutenção preventiva dos equipamentos, sendo que está previsto na legislação da ANVISA. Apenas o item que referia a existência de instrumentos ou equipamentos de medição críticos para a segurança dos alimentos apresentou adequação de 93,3\%.

Tabela 3 - Distribuição percentual geral dos SAC para cada grupo de itens do check-list. Porto Alegre, RS, 2015. 


\begin{tabular}{|c|c|c|c|c|c|}
\hline & & & & Poifu:açäa & \\
\hline & Itens avidas & 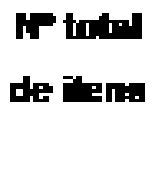 & $\begin{array}{c}\text { Adequado } \\
\text { (15i) }\end{array}$ & 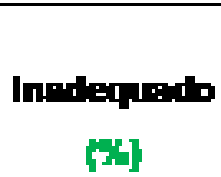 & $\begin{array}{l}\text { Hing se } \\
\text { Aplica } \\
\text { [Th] }\end{array}$ \\
\hline $\mathbf{1}$ & 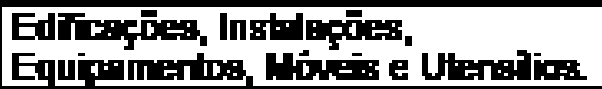 & 6 & 35,6 & 55,5 & 8,9 \\
\hline $\mathbf{2}$ & 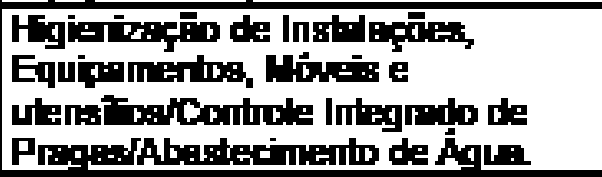 & 5 & 7B,9 & 11,1 & 10 \\
\hline$\overline{3}$ & M:Reja de Residuks & $\overline{1}$ & 53,3 & 6,7 & 0 \\
\hline 4 & 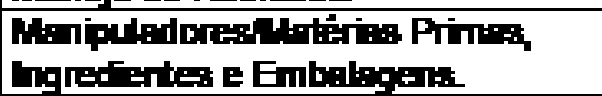 & 5 & 73,3 & $\mathbf{2 5 , 3}$ & 1,4 \\
\hline 5 & 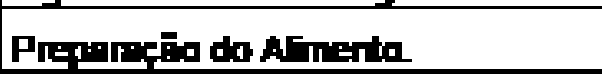 & 4 & 41,7 & 36,7 & 21,6 \\
\hline 6 & 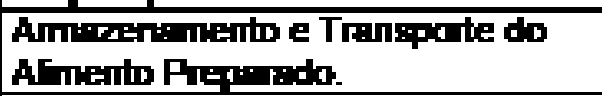 & $\overline{1}$ & 13,3 & 6,7 & BD \\
\hline $\boldsymbol{T}$ & 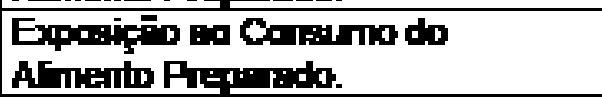 & 1 & 80 & 6,7 & 13,3 \\
\hline 8 & 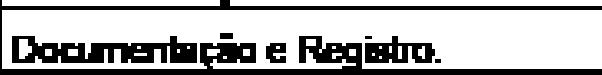 & 10 & 65,3 & 30 & 4,7 \\
\hline
\end{tabular}

A higienização de instalações, equipamentos, móveis e utensílios, controle integrado de pragas/abastecimento de água (segundo grupo) obteve $100 \%$ de adequação. Todos os restaurantes relataram utilizar saneantes registrados no Ministério da Saúde e o controle químico contra pragas e vetores urbanos são realizados por empresas especializadas. Para Mendes et al.26, na sua pesquisa, a presença de patógeno foi detectada em $38,3 \%$ das amostras de equipamentos e utensílios, sendo valores mais altos obtidos a partir de amostras do setor de distribuição, indicando a importância destes locais como fontes potenciais de transmissão de microrganismo para os alimentos. Mendes et al.27 reforça a importância de procedimentos adequados de sanitização de bancadas durante todas as etapas de processamento, para prevenir a ocorrência de surtos de doenças de origem alimentar. No estudo realizado por Menezes et al.28 também encontrou 100\% de adequação no quesito relacionado à adequação do controle de vetores e pragas urbanas.

No terceiro grupo, de manejo de resíduos, os locais também possuem coletores conforme previstos na RDC n²16/2004, onde especifica como coletores de resíduos das áreas de preparação e armazenamento de alimentos dotados de tampas acionadas sem contato manual, devidamente identificado, íntegros, sacos plásticos e em número suficiente16. Apenas um restaurante institucional público apresentou inadequação referente ao manejo de resíduos. Para Saccol et al23, o manejo de resíduos previne contra a atração, refúgio e/ou proliferação de vetores de doenças e pragas urbanas.

No item manipuladores/matérias primas, ingredientes e embalagens (grupo quatro), o único item a atingir 100\% foi referente a placas com orientações quanto à correta higienização das mãos e demais hábitos de higiene afixados em locais visíveis. Esse tipo de orientação também foi observado no estudo realizado por Rocha et al.29 em que 17 restaurantes do tipo self-service que visitou, apenas um restaurante não possuía cartazes sobre a correta higienização de mãos nas instalações sanitárias e na área de produção. Porém, a presença de orientação quanto à correta higienização das mãos não garante que será efetivamente realizado pelos manipuladores. Apesar disso, apresentou $73,3 \%$ de adequação para os locais. O papel crítico que os funcionários que lidam com alimentos em manter os consumidores seguros estão bem estabelecidos. Em um estudo de revisão realizado nos Estados Unidos da América, foi apontado que os fatores que contribuem para surtos de doenças em restaurantes estão relacionados com a origem alimentar, onde concluiu que $98 \%$ dos surtos foram causados por falta de higiene dos funcionários e/ou das práticas de preparação de alimentos 11.

Na preparação do alimento (grupo cinco) sobre registro de monitoramento de óleos e gorduras para frituras, alguns relataram não ter esse tipo de preparação, porém os que realizavam preparações com frituras (60\% dos locais), $46,6 \%$ estavam com inadequações em relação ao preenchimento dos registros. Gregório e Andrade30 avaliaram a influência do aquecimento de óleos comestíveis e concluíram que alterações físicas e químicas dos mesmos levam à formação de 
compostos que quando ingeridos podem ser prejudiciais para a saúde.

Aguarda de amostras de $100 \mathrm{~g}$ ou $100 \mathrm{~mL}$ nos restaurantes públicos foi de $80 \%$, enquanto nos privados e lanchonetes de $20 \%$ de adequação. O método de coleta de amostras é efetivo uma vez que essa medida garante que serão coletadas amostras preconizadas pela legislação. Assim, torna-se necessária para garantir análises microbiológicas dos alimentos produzidos e distribuídos em SAC tanto para controle de qualidade, como em caso de surto de DTA31.

No armazenamento e transporte do alimento preparado (grupo seis), $80 \%$ dos estabelecimentos se enquadraram na opção "não se aplica". Isso porque, devido a modalidade dos estabelecimentos, 12 dos locais não possuíam sistema de transporte dos alimentos. Os dois locais que possuíam a modalidade de transporte eram restaurantes institucionais públicos e faziam a correta verificação da temperatura dos alimentos. Apenas um local não realizava de maneira correta, pois a temperatura não era verificada no local do recebimento por não possuir instrumento de verificação da temperatura (termômetro).

Já na exposição ao consumo do alimento preparado (grupo sete), referente à existência de registro da temperatura dos equipamentos de exposição ou distribuição de alimentos preparados, verificado, datado e rubricado teve $80 \%$ de adequação, devido à maior preocupação relatada dos responsáveis técnicos serem referente à temperatura dos alimentos expostos. Porém, em um estudo realizado com manipuladores de alimentos de três países (Portugal, Sérvia e Grécia), observou-se que apenas $66,4 \%$ sabiam que as bactérias se multiplicam facilmente à temperatura ambiente. Além disso, apenas $53,7 \%$ de todos os manipuladores de alimentos tinham conhecimento que o crescimento microbiano é interrompido nas temperaturas abaixo de $-18^{\circ} \mathrm{C}$, e que as bactérias permanecem viáveis e podem crescer após 0 descongelamento, se em condições favoráveis. Em adição, apenas 40,5\% deles responderam que à temperatura de $13^{\circ} \mathrm{C}$ não é adequado para armazenamento a frio de alimentos, demonstrando assim que o conhecimento na área ainda é muito limitado32. Esses registros são importantes de serem realizados diariamente, pois em uma pesquisa realizada no município de Taubaté, São Paulo, o resultado que encontraram foi de que $87,5 \%$ dos alimentos analisados nos estabelecimentos estavam em temperaturas inadequadas, sendo os alimentos frios expostos a temperaturas acima de $10^{\circ} \mathrm{C}$ e os alimentos quentes abaixo de $60^{\circ} \mathrm{C} 34$. Mediante tal constatação, as planilhas de registro de temperatura dos equipamentos são de grande importância, pois garantem que 0 alimento seja acondicionado adequadamente e não tenha suas características sensoriais e microbiológicas alteradas $16,35$.

Por último, no grupo oito, referente à documentação e registro dos restaurantes foi encontrado um percentual de $65,3 \%$ de adequação. Bernardo et al.25, em seu estudo nos hotéis visitados em Belo Horizonte, relata que pontos importantes como documentos não estavam alinhados com a legislação vigente. No estudo realizado na cidade de São Paulo, por Saccol et. al,23 foi constatado que os itens relacionados à documentação e registro tiveram as menores taxas médias de adesão.

Já o subitem que diz respeito à implementação dos procedimentos operacionais padronizados (POPs) obrigatórios, os restaurantes públicos e privados tiveram uma média de $80 \%$ de adequação contra $40 \%$ nas lanchonetes. Todos os locais tiveram adequação nesse grupo referente ao período de 30 dias que a legislação recomenda que os registros gerados devam ser armazenados. A existência de documentos que comprovam a promoção de treinamentos para a equipe de manipuladores de alimentos do estabelecimento atingiu $60 \%$ de adequação, sendo que no estudo de Santos et al.22 ressaltaram a importância que 0 treinamento dos manipuladores como sendo a parte mais crítica de todo 0 processo de produção de alimentos, pois, eles estão ligados a todos os itens, devendo por isto, estar capacitados em relação às boas práticas de manipulação. Um estudo realizado na Finlândia mostrou que a documentação de controle foi melhor em restaurantes que tiveram uma pessoa responsável pelas questões de higiene com mais de 20 anos de experiência de trabalho no setor de alimentos, e também que a compreensão da importância da prova escrita de boas práticas parece desenvolver-se ao longo tempo33.

Assim, os registros de processos são ferramentas úteis, as quais podem ser utilizadas para o monitoramento e para a garantia da qualidade das preparações servidas, além de serem instrumentos importantes para a avaliação do desempenho e histórico dos procedimentos realizados nos SAC36. Esse estudo encontrou como principal limitação a disponibilidade da documentação completamente preenchida, pois muitas vezes alguns documentos estavam preenchidos nos locais, mas não de maneira correta e exigida pela legislação. Tal inconformidade poderia ser solucionada se fosse investido mais tempo nos estabelecimentos referente ao correto preenchimento da documentação, e também, capacitação dos funcionários envolvidos ou a seleção de um profissional habilitado para tal função. 


\section{Conclusão}

Apesar da quantidade expressiva de estudos realizados nos mais diversos tipos de restaurantes, ainda é possível observar que o grau de inadequações, muitas vezes similares nos estudos, é referente aos mesmos itens contemplados.

Os resultados apresentados nesse trabalho identificaram que a maior parte dos locais do estudo foi classificada como regular quanto ao atendimento dos itens avaliados. Apesar da visível intenção dos locais em se adequar ao que é preconizado na legislação visando à qualidade e as questões higiênico-sanitárias dos alimentos, foi identificado nesse estudo que a maior dificuldade dos SAC foi o de realizar os registros e manter a documentação em conformidade com a legislação atual.

Afora o moderado percentual de adequações encontrado nos locais, o correto preenchimento das planilhas e registros atestou a funcionalidade dos SAC e a adequação quanto às boas práticas de manipulação, a fim de evitar a ocorrência de doenças provocadas pelo consumo de alimentos contaminados.

Apesar das pesquisas atuais mostrarem a importância em manter os registros preenchidos corretamente e atualizados, muito precisa ser feito para ajudar os estabelecimentos a encontrar uma maneira de dar conta de realizá-los sem prejudicar a rotina do local. Tais medidas devem ser avaliadas e mensuradas conforme a legislação preconiza como imprescindível para a oferta de uma alimentação adequada para os seus comensais/clientes sem impor riscos à saúde.

Sugere-se que estudos dessa natureza possam ser realizados também em outras cidades, com a mesma abordagem exploratória, especialmente para comparar as realidades das diversas modalidades de SAC disponibilizados como opção do consumo de alimentos da população.

\section{Referências}

${ }^{1}$ American Dietetic Association (ADA). Position of the American Dietetic Association: domestic food and nutrition security. J Am Diet Assoc. 2002;102:1840-7.

2Cosson $\mathrm{C}$, Bolnot FH, Tronchon P. "Sécurité alimentaire" en milieu hospitalier: de la logique de crise à la logique du progrès. Nutrition Clinique et Métabolisme. 2003; 17:242-51.

3Belik W. Perspectivas para segurança alimentar e nutricional no Brasil. Rev Saúde e Sociedade. 2003;(12)1:1220.

4Santos MV, Proença RPC, Fiates GMR, Calvo MCM. Os restaurantes por peso no contexto de alimentação saudável fora de casa. Rev Nutr., Campinas. 2011; 24(4):641-9.

5Silva Júnior EA. Manual de Controle Higiênico-Sanitário em Serviços de Alimentação. São Paulo: Livraria Varela, 1995. $6^{\text {a }}$ ed. $5^{\text {a }}$ reimpressão. 2013.

6Saccol ALF. Avaliação da implementação do regulamento técnico de boas práticas para serviços de alimentação. Santa Maria: 2013. 204 p. Tese (Doutorado) - Universidade Federal de Santa Maria, 2013.

7Associação Brasileira de Empresas de Refeições Coletivas (ABERC). Acesso em 31 de maio de 2016, às 14 horas, em: http://www.aberc.com.br/mercadoreal.asp?IDMenu=21.

8Ebone MV, Cavalli SB, Lopes SJ. Segurança e qualidade higiênico-sanitária em unidades produtoras de refeições 
comerciais. Rev Nutr., Campinas. 2011; 24(5):725-34.

9Centers for Disease Control and Prevention (CDC). 2014. [citado em: 30 mar 2014]. Disponível em: http://www. cdc.gov/foodsafety/facts.html.

10Amson GV, Haracemiv SMC, Masson ML. Levantamento de dados epidemiológicos relativos à ocorrências/ surtos de doenças transmitidas por alimentos (DTAs) no estado do Paraná - Brasil, no período de 1978 a 2000. Rev Ciênc. agrotec., Lavras. 2006;30(6):1139-45.

11Shinbaum S, Crandall PG, O'Bryan CA. Evaluating your obligations for employee training according to the Food Safety Modernization Act. Rev Food Control. 2016;60:12-7.

12Welker CAD, Both JMC, Longaray SM, Haas S, Soeiro MLT, Ramos RC. Análise microbiológica dos alimentos envolvidos e surtos de doenças transmitidas por alimentos (DTA) ocorridos no estado do Rio Grande do Sul, Brasil. Rev Brasileira Bioci. 2010;8(1):44-8.

13Lupchinski CDS. Boas práticas e a categorização de serviços de alimentação para a copa FIFA 2014 no Brasil: Processo de criação e análise crítica dos critérios de avaliação.Rio Grande do Sul: 2013. Tese (Graduação) - Universidade Federal do Rio Grande do Sul. Escola de Engenharia, 2013.

14Prefeitura de Porto Alegre. Secretaria de Turismo. Acesso em 02 de Junho de 2016, às 10 horas e 30 minutos, em: http://www2.portoalegre.rs.gov.br/turismo/default.php?p_secao=258.

15Sindicato de Hospedagem e Alimentação de Poro Alegre e Região (SINDHA). Acesso em 02 de Junho de 2016, às 9 horas e 30 minutos, em: http://www.sindha.org.br/index.php/institucional/estatisticas-da-hospedagem-e-alimentacao.

16Agência Nacional de Vigilância Sanitária (ANVISA) Resolução RDC n 216/2004. Cartilha sobre boas práticas para serviços de alimentação. Realização: ANVISA e Gerência Geral de Alimentos (GGALI). Diário oficial [da] União, Poder executivo, 16 de setembro de 2004. Acesso em: http://portal.anvisa.gov.br/wps/wcm/ connect/83f33080474581508d9fdd3fbc4c6735/cartilha_gicra_final.pdf?MOD=AJPERES.

17Campos JM, Freitas APR, Silva AR. Avaliação de boas práticas de manipulação em restaurantes tipo selfservice no centro comercial de Santa Cruz do Capibaribe/PE. Rev Nutrição Brasil. 2013;12(4): 228-33.

18Secretaria da Saúde. Diário Oficial. Rio Grande do Sul. Lista de Verificação em Boas Práticas para Serviços de Alimentação - Portaria N78. Porto Alegre, 2009.

19Ribeiro-Furtini LL, Abreu LR. Utilização de APPCC na indústria de alimentos. Rev Ciênc. agrotec., Lavras. 20166;30(2):358-63.

20Agência Nacional de Vigilância Sanitária (ANVISA). Resolução RDC n²75/2002. Cartilha sobre o Regulamento Técnico de Procedimentos Operacionais Padronizados aplicados aos Estabelecimentos Produtores/Industrializadores de Alimentos. Realização: ANVISA. Diário Oficial [da] República Federativa do Brasil, Brasília, 23 de outubro de 2003. Seção 1, p. 126. Acesso em: http://www.mds.gov.br/acesso-a- informacao/legislacao/segurancaalimentar/resolucoes/2002/ Resolucao\%20RDC\%20no\%20275-\%20de\%2021\%20de\%20outubro\%20de\%202002\%20-\%20Anvisa.pdf/view

21Akutsu RC, Botelho RA, Camargo EB, Sávio KEO, Araújo WC. Adequação das boas práticas de fabricação em serviços de alimentação. Rev Nutrição, Campinas. 2005;18(3):419-27.

22Santos MOB, Rangel VP, Azeredo DP. Adequação de restaurantes comerciais às boas práticas. Rev Higiene 
sanitárias de lanchonetes. Rev Nutrire. 2013;38:438.

25Bernardo PV, Valentim ECN, Oliveira AES, Ramos SA. Avaliação das boas práticas na produção de refeições na rede hoteleira de Belo Horizonte, MG. Rev Cient Ciênc Biol Saúde. 2014;16(4):265-70.

26Mendes RA, Coelho AIM, Azeredo RMC. Contaminação por Bacillus cereus em superfícies de equipamentos e utensílios em unidade de alimentação e nutrição. Rev Ciência e Saúde Coletiva. 2011;16(9):3933-8.

27Mendes RA, Azeredo RMC, Coelho AIM, Oliveira SS, Coelho MSL. Contaminação ambiental por Bacillus cereus em unidade de alimentação e nutrição. Rev Nut., Campinas. 2004;17(2):255-61.

28Menezes JCS, Machado SGS, Campos JMC. Avaliação das boas práticas de fabricação no setor de panificação no município de Caruaru/PE. Rev Nutrição Brasil. 2014;13(3):145-9.

29Rocha B, Batista LS, Borges BM, Paiva AC. Avaliação das condições higiênico-sanitárias e da temperatura das refeições servidas em restaurantes comerciais do tipo self-service. Rev Núcleo Interdisciplinar de Pesquisa e Extensão do UNIPAM Patos de Minas: UNIPAM. 2010;7(1):30-40.

30Gregório BM, Andrade ECB. Influência do aquecimento sobre as propriedades físico-químicas de óleos comestíveis. Rev Higiene Alimentar. 2008;18(124):78-84.

31Bauer CC. Proposta de padronização de medidas caseiras para coleta de amostras em serviços de alimentação. Rio Grande do Sul, 2014. Tese (Graduação) - Universidade Federal do Rio Grande do Sul. Faculdade de Medicina, 2014.

32Smigic N, Djekic I, Martins ML, Rocha A, Sidiropoulou N, Kalogianni EP. The level of food safety knowledge in food establishments in three European countries. Rev Food Control. 2014;63:187-94.

33Läikkö-Roto T, Nevas M. Restaurant business operators' knowledge of food hygiene and their attitudes toward official food control affect the hygiene in their restaurants. Rev Food Control. 2014;43:65-73.

34Alves MG, Ueno M. Restaurantes self-service: segurança e qualidade sanitária dos alimentos servidos. Rev Nut., Campinas. 2010;23(4):573-80.

35Agência Nacional de Vigilância Sanitária (ANVISA). Material de apoio para serviços de alimentação Categorização dos Serviços de Alimentação. Brasília, 2013.

36Frantz CB, Bender B, Oliveira ABA, Tondo EC. Evaluation of production process records of fifteen food services and nutrition units. Rev Alim. Nutr., Araraquara. 2008;19(2):167-75. 